ISSN 2078-3744. Вісник Львів. ун-ту. Серія мех.-мат. 2020. Випуск 90. С. 84-91

Visnyk of the Lviv Univ. Series Mech. Math. 2020. Issue 90. P. 84-91

http://publications.lnu.edu.ua/bulletins/index.php/mmf

doi: http://dx.doi.org/10.30970/vmm.2020.90.084-091

УДК 517.537

\title{
ON THE UNIVALENCE RADII OF SUCCESSIVE GELFOND-LEONT'EV-SĂLĂGEAN AND GELFOND-LEONT'EV-RUSCHEWEYH DERIVATIVES
}

\section{Myroslav SHEREMETA}

Ivan Franko National University of Lviv, Universytetska Str., 1, 79000, Lviv, Ukraine

e-mail:m.m.sheremeta@gmail.com

For an analytic in the disk $\{z:|z|<1\}$ function $f(z)=z+\sum_{k=1}^{\infty} f_{k} z^{k}$ and formal power series $l(z)=1+\sum_{k=1}^{\infty} l_{k} z^{k}$ with $l_{k}>0$ the operator

$$
D_{l,[S] f(z)}^{n}=z+\sum_{k=2}^{\infty}\left(\frac{l_{1} l_{k-1}}{l_{k}}\right)^{n} f_{k} z^{k}
$$

is called the Gelfond-Leont'ev-Sălăgean derivative and the operator

$$
D_{l,[R]}^{n} f(z)=z+\sum_{k=2}^{\infty} \frac{l_{k-1} l_{n}}{l_{n+k-1}} f_{k} z^{k}
$$

is called the Gelfond-Leont'ev-Ruscheweyh derivative. By $\varrho[f]$ we denote the radius of the univalence of the function $f$. It is proved, for example, that for each $n \geq 1$

$$
\frac{\sqrt{2}-1}{\sqrt{2}}\left|\frac{f_{1}}{f_{2}}\right|\left(\frac{l_{2}}{l_{1}^{2}}\right)^{n} \leq \varrho\left[D_{l,[S]}^{n} f\right] \leq 2\left|\frac{f_{1}}{f_{2}}\right|\left(\frac{l_{2}}{l_{1}^{2}}\right)^{n}
$$

and

$$
\frac{\sqrt{2}-1}{\sqrt{2}}\left|\frac{f_{1}}{f_{2}}\right| \frac{l_{n+1}}{l_{1} l_{n}} \leq \varrho\left[D_{l,[R]}^{n} f\right] \leq 2\left|\frac{f_{1}}{f_{2}}\right| \frac{l_{n+1}}{l_{1} l_{n}} .
$$

Key words: analytic function, Gelfond-Leont'ev-Sălăgean derivative, Gelfond-Leont'ev-Ruscheweyh derivative, radius of the univalence.

2020 Mathematics Subject Classification: 30D99

(C) Sheremeta, M., 2020 


\section{INTRODUCTION}

For a formal power series

$$
f(z)=f_{0}+\sum_{k=1}^{\infty} f_{k} z^{k}, \quad z=r e^{i \theta},
$$

and $l(z)=1+\sum_{k=1}^{\infty} l_{k} z^{k}\left(l_{k}>0\right)$ the formal power series $D_{l}^{n} f(z)=\sum_{k=0}^{\infty} \frac{l_{k}}{l_{k+n}} f_{k+n} z^{k}$ is called the Gelfond-Leont'ev derivative [1]. If $l(z)=e^{z}$ (i.e. $\left.l_{k}=1 / k !\right)$ then $D_{l}^{n} f=f^{(n)}$ is the usual derivative.

If the function $f(z)=z+\sum_{k=2}^{\infty} f_{k} z^{k}$ is analytic in the disk $\{z:|z|<1\}$ then the operator $D_{[S]}^{n} f(n \geq 0)$ defined by

$$
\begin{gathered}
D_{[S]}^{0} f(z)=f(z), \quad D_{[S]}^{1} f(z)=D_{[S]} f(z)=z f^{\prime}(z), \\
D_{[S]}^{n} f(z)=D_{[S]}\left(D_{[S]}^{n-1} f(z)\right)=z+\sum_{k=2}^{\infty} k^{n} f_{k} z^{k}
\end{gathered}
$$

is known as the Sălăgean derivative $[2]$. The operator

$$
D_{[R]}^{n} f(z)=\frac{z}{n !} \frac{d^{n}}{d z^{n}}\left\{z^{n-1} f(z)\right\}=z+\sum_{k=2}^{\infty} \frac{(k+n-1) !}{n !(k-1) !} f_{k} z^{k}
$$

is called $[3]$ the Ruscheweyh derivative.

In [4], combining the definitions of Gelfond-Leont'ev derivative with Sălăgean derivative and Ruscheweyh derivative, the operator

$$
D_{l,[S]}^{n} f(z)=l_{1} z D_{l}^{1}\left(D_{l,[S]}^{n-1} f(z)\right)=z+\sum_{k=2}^{\infty}\left(\frac{l_{1} l_{k-1}}{l_{k}}\right)^{n} f_{k} z^{k}
$$

is called the Gelfond-Leont'ev-Sălăgean derivative and the operator

$$
D_{l,[R]}^{n} f(z)=z l_{n} D_{l}^{n}\left\{z^{n-1} f(z)\right\}=z+\sum_{k=2}^{\infty} \frac{l_{k-1} l_{n}}{l_{n+k-1}} f_{k} z^{k}
$$

is called the Gelfond-Leont'ev-Ruscheweyh derivative. In [4 the behavior of maximal terms of successive Gelfond-Leont'ev-Sălăgen and Gelfond-Leont'ev-Ruscheweyh derivatives and in $[5]$ the properties of Hadamard compositions of such derivatives are studied.

The radius $\varrho[f]$ of univalence of a function $f$ is defined as follows: if $f^{\prime}(0)=0$ then we put $\varrho[f]=0$, and if $f^{\prime}(0) \neq 0$ then $\varrho[f]$ is a radius of the largest disk with the center at a point $z=0$, in which the function $f$ is univalent. The asymptotic behavior of the sequence of radii of the univalence of ordinary derivatives of the function $f$ has been studied by many authors. The most significant contribution was made by S.M. Shah and S.Y. Trimble [6] 8]. The asymptotic behavior of the sequence of radii of the univalence of Gelfond-Leont'ev derivatives is investigated in [9-12].

Here we consider a similar problem for Gelfond-Leont'ev-Sălăgen and GelfondLeont'ev-Ruscheweyh derivatives. Our research is based on the following lemmas [11]. 
Lemma 1. Let $\alpha(z)=\sum_{k=0}^{\infty} \alpha_{k} z^{k}$ and $\varrho \in(0,+\infty)$. If the function $\alpha$ is univalent in $\mathbb{D}_{\varrho}=\{z:|z|<\varrho\}$ then $\left|\alpha_{k}\right| \varrho^{k-1} \leq k\left|\alpha_{1}\right|$ for all $k \geq 1$.

Lemma 2. Let $\alpha(z)=\sum_{k=0}^{\infty} \alpha_{k} z^{k}$. If $\sum_{k=2}^{\infty} k\left|\alpha_{k}\right| \varrho^{k-1} \leq\left|\alpha_{1}\right|$ for some $\varrho \in(0,+\infty)$ then the function $\alpha$ is univalent in $\mathbb{D}_{\varrho}$.

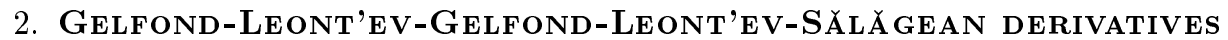

Here for a function (1) we will consider a more general form of the Gelfond-Leont'evSălăgean derivative $D_{l,[S]}^{n} f$.

We remark that if a function $f$ is given by a gap power series

$$
f(z)=f_{0}+\sum_{j=0}^{\infty} f_{k_{j}+1} z^{k_{j}+1}, \quad f_{k_{j}+1} \neq 0(j \geq 0),
$$

where $0 \leq k_{j} \uparrow \infty$ as $0 \leq j \rightarrow \infty$, then

$$
D_{l,[S]}^{k_{n}} f(z)=\sum_{j=0}^{\infty}\left(\frac{l_{1} l_{k_{j}}}{l_{k_{j}+1}}\right)^{k_{n}} f_{k_{j}+1} z^{k_{j}+1} .
$$

Theorem 1. If the function $f$ is given by a gap power series (2) then for the radius of univalence of Gelfond-Leont'ev-Sălăgean derivative $D_{l,[S]}^{k_{n}} f$ the estimates

(4)

$$
\frac{\sqrt{2}-1}{\sqrt{2}} \sqrt[k_{j}]{\mid \frac{f_{k_{0}+1}}{f_{k_{j}+1} \mid}}\left(\frac{l_{k_{0}} l_{k_{j}+1}}{l_{k_{j}} l_{k_{0}+1}}\right)^{k_{n} / k_{j}} \leq \varrho\left[D_{l,[S]}^{k_{n}} f\right] \leq \sqrt[k_{j}]{\left(k_{j}+1\right)\left|\frac{f_{k_{0}+1}}{f_{k_{j}+1}}\right|}\left(\frac{l_{k_{0}} l_{k_{j}+1}}{l_{k_{j}} l_{k_{0}+1}}\right)^{k_{n} / k_{j}}
$$

hold for every $j \geq 1$.

Proof. If function $(3)$ is univalent in $\mathbb{D}_{\varrho}$ then by Lemma 1

$$
\left|\left(\frac{l_{1} l_{k_{j}}}{l_{k_{j}+1}}\right)^{k_{n}} f_{k_{j}+1}\right| \varrho^{k_{j}} \leq\left(k_{j}+1\right)\left(\frac{l_{1} l_{k_{0}}}{l_{k_{0}+1}}\right)^{k_{n}}\left|f_{k_{0}+1}\right|,
$$

i.e.

$$
\varrho\left[D_{l,[S]}^{k_{n}} f\right]^{k_{j}} \leq\left(k_{j}+1\right)\left|\frac{f_{k_{0}+1}}{f_{k_{j}+1}}\right|\left(\frac{l_{k_{0}} l_{k_{j}+1}}{l_{k_{j}} l_{k_{0}+1}}\right)^{k_{n}},
$$

whence we obtain the right hand side of (4).

Now let $0<x \leq(\sqrt{2}-1) / \sqrt{2}$. Then

$$
\sum_{j=1}^{\infty}\left(k_{j}+1\right) x^{k_{j}} \leq \sum_{j=1}^{\infty}(j+1) x^{j}=\frac{1}{(1-x)^{2}}-1 \leq 1 .
$$

Therefore, if

$$
\varrho=\frac{\sqrt{2}-1}{\sqrt{2}} \sqrt[k_{j}]{\left|\frac{f_{k_{0}+1}}{f_{k_{j}+1}}\right|}\left(\frac{l_{k_{0}} l_{k_{j}+1}}{l_{k_{j}} l_{k_{0}+1}}\right)^{k_{n} / k_{j}}
$$


then

$$
x=\varrho \sqrt[k_{j}]{\left|\frac{f_{k_{j}+1}}{f_{k_{0}+1}}\right|}\left(\frac{l_{k_{j}} l_{k_{0}+1}}{l_{k_{0}} l_{k_{j}+1}}\right)^{k_{n} / k_{j}}=\frac{\sqrt{2}-1}{\sqrt{2}}
$$

and, thus,

$$
\sum_{j=1}^{\infty}\left(k_{j}+1\right)\left(\frac{l_{k_{j}} l_{k_{0}+1}}{l_{k_{0}} l_{k_{j}+1}}\right)^{k_{n}}\left|\frac{f_{k_{j}+1}}{f_{k_{0}+1}}\right| \varrho^{k_{j}}=\sum_{j=1}^{\infty}\left(k_{j}+1\right) x^{k_{j}} \leq 1
$$

whence

$$
\sum_{j=1}^{\infty}\left(k_{j}+1\right)\left(\frac{l_{1} l_{k_{j}}}{l_{k_{j}+1}}\right)^{k_{n}}\left|f_{k_{j}+1}\right| \varrho^{k_{j}} \leq\left(\frac{l_{1} l_{k_{0}}}{l_{k_{0}+1}}\right)^{k_{n}}\left|f_{k_{0}+1}\right| .
$$

Hence by Lemma 2 the function $D_{l,[S] f}^{k_{n}}$ is univalent in $\mathbb{D}_{\varrho}$ and, thus, in view of

$$
\varrho\left[D_{l,[S]}^{k_{n}} f\right] \geq \frac{\sqrt{2}-1}{\sqrt{2}} \sqrt[k_{j}]{\mid \frac{f_{k_{0}+1}}{f_{k_{j}+1} \mid}}\left(\frac{l_{k_{0}} l_{k_{j}+1}}{l_{k_{j}} l_{k_{0}+1}}\right)^{k_{n} / k_{j}}
$$

for every $j \geq 1$, i.e. the left hand side of $(4)$ is correct. Theorem 1 is proved.

If series (2) has a radius of convergence $R \in(0,+\infty)$ then

$$
\varliminf_{j \rightarrow \infty} \sqrt[k_{j}]{\left(k_{j}+1\right)\left|\frac{f_{k_{0}+1}}{f_{k_{j}+1}}\right|}=R
$$

and, since $\lim _{j \rightarrow \infty} a_{j} b_{j} \leq \lim _{j \rightarrow \infty} a_{j} \varlimsup_{j \rightarrow \infty} b_{j}$, from (4) we get

$$
\varrho\left[D_{l,[S]}^{k_{n}} f\right] \leq \varliminf_{j \rightarrow \infty} \sqrt[k_{j}]{\left(k_{j}+1\right)\left|\frac{f_{k_{0}+1}}{f_{k_{j}+1}}\right|}\left(\frac{l_{k_{0}} l_{k_{j}+1}}{l_{k_{j}} l_{k_{0}+1}}\right)^{k_{n} / k_{j}} \leq R\left(\varlimsup_{j \rightarrow \infty} \sqrt[k_{j}]{\frac{l_{k_{0}} l_{k_{j}+1}}{l_{k_{j}} l_{k_{0}+1}}}\right)^{k_{n}} .
$$

Similarly, since $\varlimsup_{j \rightarrow \infty} a_{j} b_{j} \geq \underset{j \rightarrow \infty}{\lim _{j \rightarrow \infty}} a_{j} \varlimsup_{j \rightarrow \infty} b_{j}$, from (6) we get

$\varrho\left[D_{l,[S]}^{k_{n}} f\right] \geq \frac{\sqrt{2}-1}{\sqrt{2}} \varlimsup_{j \rightarrow \infty} \sqrt[k_{j}]{\left|\frac{f_{k_{0}+1}}{f_{k_{j}+1}}\right|}\left(\frac{l_{k_{0}} l_{k_{j}+1}}{l_{k_{j}} l_{k_{0}+1}}\right)^{k_{n} / k_{j}} \geq \frac{\sqrt{2}-1}{\sqrt{2}} R\left(\varlimsup_{j \rightarrow \infty} \sqrt[k_{j}]{\frac{l_{k_{0}} l_{k_{j}+1}}{l_{k_{j}} l_{k_{0}+1}}}\right)^{k_{n}}$.

Therefore, we obtain the following statement.

Proposition 1. If a function $f$ is given by the gap power series (2) with the radius of convergence $R \in(0,+\infty)$ then for the radius of univalence of Gelfond-Leont'ev-Sălăgean derivative $D_{l,[S]}^{k_{n}} f$ the estimates

$$
\frac{\sqrt{2}-1}{\sqrt{2}} R\left(\varlimsup_{j \rightarrow \infty} \sqrt[k_{j}]{\frac{l_{k_{0}} l_{k_{j}+1}}{l_{k_{j}} l_{k_{0}+1}}}\right)^{k_{n}} \leq \varrho\left[D_{l,[S]}^{k_{n}} f\right] \leq R\left(\varlimsup_{j \rightarrow \infty} \sqrt[k_{j}]{\frac{l_{k_{0}} l_{k_{j}+1}}{l_{k_{j}} l_{k_{0}+1}}}\right)^{k_{n}}
$$

hold.

Theorem 1 and Proposition 1 imply the following statement. 
Corollary 1. For the radius of univalence of Gelfond-Leont'ev-Sălăgean derivative $D_{l,[S]}^{n} f$ of function (1) the estimates

$$
\frac{\sqrt{2}-1}{\sqrt{2}} \sqrt[j]{\left|\frac{f_{1}}{f_{j+1}}\right|}\left(\frac{l_{j+1}}{l_{j} l_{1}}\right)^{n / j} \leq \varrho\left[D_{l,[S]}^{n} f\right] \leq \sqrt[j]{(j+1)\left|\frac{f_{1}}{f_{j+1}}\right|}\left(\frac{l_{j+1}}{l_{j} l_{1}}\right)^{n / j}
$$

hold for every $j \geq 1$. If, moreover, series (1) has the radius of convergence $R \in(0,+\infty)$ then

$$
\frac{\sqrt{2}-1}{\sqrt{2}} R\left(\varlimsup_{j \rightarrow \infty} \sqrt[j]{\frac{l_{j+1}}{l_{j}}}\right)^{n} \leq \varrho\left[D_{l,[S]}^{n} f\right] \leq R\left(\varlimsup_{j \rightarrow \infty} \sqrt[j]{\frac{l_{j+1}}{l_{j}}}\right)^{n} .
$$

We remark also that for $j=1$ from (7) we get

$$
\frac{\sqrt{2}-1}{\sqrt{2}}\left|\frac{f_{1}}{f_{2}}\right|\left(\frac{l_{2}}{l_{1}^{2}}\right)^{n} \leq \varrho\left[D_{l,[S]}^{n} f\right] \leq 2\left|\frac{f_{1}}{f_{2}}\right|\left(\frac{l_{2}}{l_{1}^{2}}\right)^{n} .
$$

\section{Gelfond-Leont'ev-Ruscheweyh Derivatives}

Here for a function (1) we will consider also a slightly more general form of the Gelfond-Leont'ev-Ruscheweyh derivative

$$
D_{l,[R]}^{n}(z)=\sum_{k=1}^{\infty} \frac{l_{k-1} l_{n}}{l_{n+k-1}} f_{k} z^{k} .
$$

We remark that if a function $f$ is given by a gap power series $(2)$ then

$$
D_{l,[R]}^{k_{n}}(z)=\sum_{j=0}^{\infty} \frac{l_{k_{n}} l_{k_{j}}}{l_{k_{j}+k_{n}}} f_{k_{j}+1} z^{k_{j}+1} .
$$

Theorem 2. If a function $f$ is given by a gap power series (2) then for the radius of univalence of Gelfond-Leont'ev-Ruscheweyh derivative $D_{l,[R]}^{k_{n}} f$ the estimates

$$
\frac{\sqrt{2}-1}{\sqrt{2}} \sqrt[k_{j}]{\left|\frac{f_{k_{0}+1}}{f_{k_{j}+1}}\right| \frac{l_{k_{0}} l_{k_{j}+k_{n}}}{l_{k_{j}} l_{k_{0}+k_{n}}}} \leq \varrho\left[D_{l,[R]}^{k_{n}} f\right] \leq \sqrt[k_{j}]{\left(k_{j}+1\right)\left|\frac{f_{k_{0}+1}}{f_{k_{j}+1}}\right| \frac{l_{k_{0}} l_{k_{j}+k_{n}}}{l_{k_{j}} l_{k_{0}+k_{n}}}}
$$

hold for every $j \geq 1$.

Proof. If the function $(8)$ is univalent in $\mathbb{D}_{\varrho}$ then by Lemma 1

$$
\left|\frac{l_{k_{n}} l_{k_{j}}}{l_{k_{j}+k_{n}}} f_{k_{j}+1}\right| \varrho^{k_{j}} \leq\left(k_{j}+1\right) \frac{l_{k_{n}} l_{k_{0}}}{l_{k_{0}+k_{n}}}\left|f_{k_{0}+1}\right|,
$$

i.e.

$$
\varrho\left[D_{l,[R]}^{k_{n}} f\right]^{k_{j}} \leq\left(k_{j}+1\right)\left|\frac{f_{k_{0}+1}}{f_{k_{j}+1}}\right| \frac{l_{k_{j}+k_{n}} l_{k_{0}}}{l_{k_{0}+k_{n}} l_{k_{j}}}
$$

whence we obtain the right hand side of (9).

Now we put

$$
\varrho=\frac{\sqrt{2}-1}{\sqrt{2}} \sqrt[k_{j}]{\left|\frac{f_{k_{0}+1}}{f_{k_{j}+1}}\right| \frac{l_{k_{0}} l_{k_{j}+k_{n}}}{l_{k_{j}} l_{k_{0}+k_{n}}}} .
$$


Then as in the proof of Theorem 1 we obtain

$$
\sum_{j=1}^{\infty}\left(k_{j}+1\right) \frac{l_{k_{n}} l_{k_{j}}}{l_{k_{j}+k_{n}}}\left|f_{k_{j}+1}\right| \varrho^{k_{j}} \leq \frac{l_{k_{n}} l_{k_{0}}}{l_{k_{0}+k_{n}}}\left|f_{k_{0}+1}\right| .
$$

Hence by Lemma 2 the function $D_{l,[R] f}^{k_{n}}$ is univalent in $\mathbb{D}_{\varrho}$ and, thus, in view of 10

$$
\varrho\left[D_{l,[R] f}^{k_{n}}\right] \geq \frac{\sqrt{2}-1}{\sqrt{2}} \sqrt[k_{j}]{\left|\frac{f_{k_{0}+1}}{f_{k_{j}+1}}\right| \frac{l_{k_{0}} l_{k_{j}+k_{n}}}{l_{k_{j}} l_{k_{0}+k_{n}}}}
$$

for every $j \geq 1$, i.e. the left hand side of $(9)$ is correct. Theorem 2 is proved.

Using (9), 11 and repeating the proof of Proposition 1 we come to the next statement.

Proposition 2. If a function $f$ is given by the gap power series (2) with the radius of convergence $R \in(0,+\infty)$ then for the radius of univalence of Gelfond-Leont'evRuscheweyh derivative $D_{l,[R]}^{k_{n}} f$ the following estimates we have

$$
\frac{\sqrt{2}-1}{\sqrt{2}} R \varlimsup_{j \rightarrow \infty} \sqrt[k_{j}]{\frac{l_{k_{0}} l_{k_{j}+k_{n}}}{l_{k_{j}} l_{k_{0}+k_{n}}}} \leq \varrho\left[D_{l,[R]}^{k_{n}} f\right] \leq R \varlimsup_{j \rightarrow \infty} \sqrt[k_{j}]{\frac{l_{k_{0}} l_{k_{j}+k_{n}}}{l_{k_{j}} l_{k_{0}+k_{n}}}} .
$$

Theorem 2 and Proposition 2 imply the following statement.

Corollary 2. For the radius of univalence of Gelfond-Leont'ev-Ruscheweyh derivative $D_{l,[R]}^{n} f$ of a function (1) the estimates

$$
\frac{\sqrt{2}-1}{\sqrt{2}} \sqrt[j]{\left|\frac{f_{1}}{f_{j+1}}\right| \frac{l_{j+n}}{l_{j} l_{n}}} \leq \varrho\left[D_{l,[R]}^{n} f\right] \leq \sqrt[j]{(j+1)\left|\frac{f_{1}}{f_{j+1}}\right| \frac{l_{j+n}}{l_{j} l_{n}}}
$$

hold for every $j \geq 1$. If, moreover, series (1) has the radius of convergence $R \in(0,+\infty)$ then

$$
\frac{\sqrt{2}-1}{\sqrt{2}} R \varlimsup_{j \rightarrow \infty} \sqrt[j]{\frac{l_{j+n}}{l_{j} l_{n}}} \leq \varrho\left[D_{l,[S]}^{n} f\right] \leq R \varlimsup_{j \rightarrow \infty} \sqrt[j]{\frac{l_{j+n}}{l_{j} l_{n}}} .
$$

For $j=1$ from $(12)$ we get

$$
\frac{\sqrt{2}-1}{\sqrt{2}}\left|\frac{f_{1}}{f_{2}}\right| \frac{l_{n+1}}{l_{1} l_{n}} \leq \varrho\left[D_{l,[R]}^{n} f\right] \leq 2\left|\frac{f_{1}}{f_{2}}\right| \frac{l_{n+1}}{l_{1} l_{n}} .
$$

Choosing a function $l$ in one or another way, we obtain the corresponding estimates for the radius of univalence.

Example 1. Let $l_{k}=\exp \left\{a k^{2}\right\}$. Then $\frac{l_{j+1}}{l_{j}}=\exp \{2 a j+1\}$ and $\frac{l_{j+n}}{l_{j} l_{n}}=\exp \{2 a j n\}$. Therefore, if series (1) has the radius of convergence $R \in(0,+\infty)$ then by Corollaries 1 and 2

$$
\frac{\sqrt{2}-1}{\sqrt{2}} R e^{2 a n} \leq \varrho\left[D_{l,[S]}^{n} f\right], \varrho\left[D_{l,[R]}^{n} f\right] \leq R e^{2 a n} .
$$


Example 2. If $l_{k}=q^{k}$ then $\frac{l_{j+1}}{l_{j}}=q$ and $\frac{l_{j+n}}{l_{j} l_{n}}=1$. Therefore, if series (1) has the radius of convergence $R \in(0,+\infty)$ then

$$
\frac{\sqrt{2}-1}{\sqrt{2}} R \leq \varrho\left[D_{l,[S]}^{n} f\right], \varrho\left[D_{l,[R]}^{n} f\right] \leq R .
$$

Example 3. If $l_{k}=\frac{1}{k !}$ then $\frac{l_{j+1}}{l_{j}}=\frac{1}{j+1}$ and $\frac{l_{j+n}}{l_{j} l_{n}}=\frac{j ! n !}{(j+n) !}$. Therefore, if series (1) has the radius of convergence $R \in(0,+\infty)$ then estimates 13$)$ hold.

\section{REFERENCES}

1. A. O. Gel'fond and A. F. Leont'ev, On a generalization of Fourier series, Mat. Sb. (N.S.), 29(71) (1951), no. 3, 477-500 (in Russian).

2. G. St. Sălăgean, Subclasses of univalent functions, In: C. A. Cazacu, N. Boboc, M. Jurchescu, I. Suciu (eds), Complex Analysis - Fifth Romanian-Finnish Seminar. Lecture Notes in Mathematics, vol 1013. Springer, Berlin, Heidelberg. 1983, pp. 362-372.

DOI: $10.1007 /$ BFb0066543

3. St. Ruscheweyh, New criteria for univalent functions, Proc. Amer. Math. Soc. 49 (1975), no. 1, 109-115. DOI: 10.1090/S0002-9939-1975-0367176-1

4. M. M. Sheremeta, On the maximal terms of successive Gelfond-Leont'ev-Sălăgean and Gelfond-Leont'ev-Ruscheweyh derivatives of a function analytic in the unit disc, Mat. Stud. 37 (2012), no. 1, 58-64.

5. M. M. Sheremeta, Hadamard composition of Gelfond-Leont'ev-Sălăgean and Gelfond-Leont'ev-Ruscheweyh derivatives of functions analytic in the unit disc, Mat. Stud. 54 (2020), no. 2, 115-134. DOI: $10.30970 / \mathrm{ms} .54 .2 .115-134$

6. S. M. Shah and S. Y. Trimble, Univalent functions with univalent derivatives, II, Trans. Amer. Math. Soc. 144 (1969), 313-320. DOI: 10.2307/1995283

7. S. M. Shah and S. Y. Trimble, Univalence of derivatives of functions defined by gap power series, J. London. Math. Soc. (2) 9 (1975), no. 3, 501-512. DOI: 10.1112/jlms/s2-9.3.501

8. S. M. Shah and S. Y. Trimble, Univalence of derivatives of functions defined by gap power series, II, J. Math. Anal. Appl. 56 (1976), no. 1, 28-40. DOI: 10.1016/0022-247X(76)90005-6

9. G. P. Kapoor, O. P. Juneja, and J. Patel, Univalence of Gelfond-Leont'ev derivatives of analytic functions, Bull. Math. Soc. Sci. Math. Répub. Soc. Roum., Nouv. Sér. 33 (1989), no. $1,25-34$.

10. G. P. Kapoor and J. Patel, Univalence of Gelfond-Leont'ev derivatives of functions defined by gap power series, Rend. Mat. Appl., VII. Ser. 6 (1986), no. 4, 491-502.

11. М. М. Шеремета, Про радіуси однолистості похідних Гелъбонда-Леонтъева, Укр. мат. ж. 47 (1995), nо. 3, 390-399; English version: M. M. Sheremeta, On the univalence radii of Gelfond-Leont'ev derivatives, Ukr. Math. J. 47 (1995), no. 3, 454-464 DOI: 10.1007/BF01056307

12. O. Volokh and M. Sheremeta, On the univalence radii of Gelfond-Leont'ev derivatives of gap power series, Visnyk Lviv Univ. Ser. Mech.-Math. 68 (2008), 59-67 (in Ukrainian).

Статтл: надійшла до редколегї 03.10.2020 прийнята до друку 17.11.2021 
ON THE UNIVALENCE RADII OF SUCCESSIVE ...

\section{ПРО РАДІУСИ ОДНОЛИСТОСТІ ПОСЛІДОВНИХ ПОХІДНИХ ГЕЛЬФОНДА-ЛЕОНТЬЕВА-САЛАГЕНА I ГЕЛЬФОНДА-ЛЕОНТЬЕВА-РУШЕВЕЯ}

\section{Мирослав Шеремета}

Лъвівсъкий націоналъний університет імені Івана Франка, вул. Університетсъка, 1, 79000, Лъвів

e-mail: m.m.sheremeta@gmail.com

Для аналітичної в крузі $\{z:|z|<1\}$ функції $f(z)=z+\sum_{k=1}^{\infty} f_{k} z^{k}$ i формального степеневого ряду $l(z)=1+\sum_{k=1}^{\infty} l_{k} z^{k}$ з $l_{k}>0$ оператор $D_{l,[S]}^{n} f(z)=z+\sum_{k=2}^{\infty}\left(\frac{l_{1} l_{k-1}}{l_{k}}\right)^{n} f_{k} z^{k}$ називається похідною ГельфондаЛеонтьєва-Салагена, а оператор $D_{l,[R]}^{n} f(z)=z+\sum_{k=2}^{\infty} \frac{l_{k-1} l_{n}}{l_{n+k-1}} f_{k} z^{k}$ називається похідною Гельфонда-Леонтьєва-Рушевея. Через $\varrho[f]$ позначимо радіус однолистості функції $f$. Доведено, наприклад, що для кожного $n \geq 1$

$$
\frac{\sqrt{2}-1}{\sqrt{2}}\left|\frac{f_{1}}{f_{2}}\right|\left(\frac{l_{2}}{l_{1}^{2}}\right)^{n} \leq \varrho\left[D_{l,[S]}^{n} f\right] \leq 2\left|\frac{f_{1}}{f_{2}}\right|\left(\frac{l_{2}}{l_{1}^{2}}\right)^{n}
$$

$$
\frac{\sqrt{2}-1}{\sqrt{2}}\left|\frac{f_{1}}{f_{2}}\right| \frac{l_{n+1}}{l_{1} l_{n}} \leq \varrho\left[D_{l,[R]}^{n} f\right] \leq 2\left|\frac{f_{1}}{f_{2}}\right| \frac{l_{n+1}}{l_{1} l_{n}} .
$$

Ключові слова: аналітична функція, похідна Гельфонда-ЛеонтьєваСалагена, похідна Гельфонда-Леонтьєва-Рушевея, радіус однолистості. 